\title{
Everyday Cognitive Failure in Sarcoidosis: The Prevalence and the Effect of Anti-TNF- $\alpha$ Treatment
}

\author{
Marjon D. Elfferich ${ }^{a-c}$ Patty J. Nelemans ${ }^{a, f}$ Rudolf W. Ponds ${ }^{a, d}$ \\ Jolanda De Vries ${ }^{\mathrm{a}, \mathrm{g}}$ Petal A. Wijnen ${ }^{\mathrm{a}, \mathrm{e}}$ Marjolein Drent ${ }^{\mathrm{a}, \mathrm{c}}$ \\ ${ }^{\mathrm{a}}$ ild care center and Departments of ${ }^{\mathrm{b}}$ Physical Therapy, ${ }^{\mathrm{c}}$ Respiratory Medicine, ${ }^{\mathrm{d}}$ Psychiatry and Psychology, \\ and ${ }^{e}$ Clinical Chemistry, Maastricht University Medical Center, and ${ }^{f}$ Department of Epidemiology, University of \\ Maastricht, Maastricht, and ${ }^{9}$ Departments of Medical Psychology, CoRPS, Tilburg University, and St. Elisabeth \\ Hospital, Tilburg, The Netherlands
}

\section{Key Words}

Anti-TNF- $\alpha$ therapy $\cdot$ Cognitive function $\cdot$

Neuropsychological deficits · Sarcoidosis · Adalimumab • Infliximab

\begin{abstract}
Background: Cognitive symptoms, such as concentration problems, are frequently recorded by sarcoidosis patients. Objectives: The aim of this study was to assess the prevalence of perceived everyday cognitive failure in sarcoidosis patients and healthy controls. Furthermore, the effect of treatment on cognitive functioning was examined. Methods: The study included 343 sarcoidosis patients $(44.6 \% \mathrm{fe}-$ males; age $49.3 \pm 11.0$ years). They completed the Cognitive Failure Questionnaire (CFQ) and Fatigue Assessment Scale (FAS) at baseline and the 6-month follow-up to evaluate the effect of treatment on cognitive functioning. The control group consisted of 343 age- and sex-matched healthy controls. Results: The mean CFQ score was significantly higher in sarcoidosis patients $(37.3 \pm 16.1)$ compared with the controls $(31.3 \pm 10.1 ; p<0.0001)$. A high CFQ sore $(\geq 43)$ was found in $35.0 \%$ of the patients and only $14.3 \%$ of the controls. No relation with disease severity and duration, or disease lo-
\end{abstract}

\section{KARGER}

Fax +41613061234 E-Mail karger@karger.ch www.karger.com
(C) 2010 S. Karger AG, Basel

0025-7931/10/0803-0212\$26.00/0

Accessible online at:

www.karger.com/res cation was found. The proportion of patients receiving treatment did not differ among the groups with high and normal CFQ score. At the 6-month follow-up, only patients recently treated with anti-TNF- $\alpha$ therapy $(n=42)$ demonstrated a significant improvement in the CFQ score $(\Delta-7.07 \pm 7.23)$ compared with the untreated patients $(\Delta-0.08 \pm 9.35)$ and patients treated with prednisone with or without methotrexate $(\Delta 1.67 \pm 9.22 ; \mathrm{p}<0.0001)$. After adjustment for the concomitant decrease in fatigue, the effect of anti-TNF- $\alpha$ therapy remained high and significant. Conclusions: Subjective cognitive failure is a substantial problem in sarcoidosis patients regardless of disease severity. Anti-TNF- $\alpha$ therapy had a positive effect on cognition, fatigue and other symptoms of sarcoidosis.

Copyright $\odot 2010$ S. Karger AG, Basel

\section{Introduction}

Cognitive impairment, characterized by memory loss and concentration problems, has been observed in several chronic disorders in previous studies. Severe chronic obstructive pulmonary disease was associated with lower cognitive performance over time [1]. Patients suffering

Tel. +31 43387 6497, Fax +31 43387 4718, E-Mail m.drent@mumc.nl 
from sarcoidosis often report cognitive complaints, such as memory loss, concentration problems and other mental problems. However, up to now no studies have been performed to assess this problem extensively in sarcoidosis.

Sarcoidosis is an inflammatory disease with a high incidence among young adults. The clinical course of sarcoidosis is highly variable [2-5]. A spontaneous remission may occur or it may become chronic. Patients often present with symptoms that do not correspond with objective physical evidence. Patients with pulmonary sarcoidosis may present with symptoms related directly to the chest, such as cough, dyspnea on exertion, retrosternal chest pain, chest discomfort and wheezing. Fever, anorexia, weight loss, general weakness, fatigue, pain, mental problems and reduced quality of life are also associated with sarcoidosis [6-8]. These symptoms are disabling, particularly when they become chronic. Previously, we found that sarcoidosis patients with current symptoms displayed more depressive symptoms and health status scores were lower compared with patients without current symptoms [7]. In line with this finding, Cox et al. [9] found that higher scores on depressive symptoms and perceived stress were related to lower health status scores in sarcoidosis. Furthermore, Chang et al. [10] found a prevalence of depression of $60 \%$ in their sarcoidosis population.

Broadbent et al. [11] described 'cognitive failure' as a cognitive error that occurs during the performance of a task that a person normally would execute successfully. They developed the Cognitive Failure Questionnaire (CFQ, see Appendix), a self-report questionnaire assessing failures in everyday errors of attention, perception, memory and motor function [11]. In general, the CFQ appears to be a reliable and brief measure useful in clinical practice.

The aim of this study was to assess the prevalence of perceived cognitive problems using the CFQ in patients with sarcoidosis compared with healthy controls. Moreover, within a subpopulation of these patients, perceived cognitive functioning levels evaluated at baseline and after 6 months of treatment were compared. The effect of different types of treatment initiated for sarcoidosis-related problems, but not cognitive failure, were also assessed using the CFQ.

\section{Patients and Methods}

Sarcoidosis patients $(n=441)$ attending the outpatient clinic of the Department of Respiratory Medicine of the Maastricht University Medical Center were sent a set of questionnaires in November 2007. Exclusion criteria were poor knowledge of the
Dutch language $(\mathrm{n}=4)$ and co-morbidity, such as a malignancy $(\mathrm{n}=24)$. The diagnosis was based on clinical features consistent with sarcoidosis, according to the guidelines of the World Association of Sarcoidosis and Other Granulomatous Diseases [12]. Biopsies were available in $80 \%$ of cases; no biopsy was obtained in patients with Löfgren's syndrome.

Of the 441 patients, $343(77.8 \%)$ returned the questionnaires and were included in the study. To assess the effect of treatment on cognition, the questionnaires were completed for the second time 6 months after baseline $(324 / 343=94.5 \%$ of cases $)$. Demographic and medical data of all participants were obtained. Healthy volunteers $(n=1,357)$, who participated in the Maastricht Aging Study, served as a sex- and age-matched control group $(\mathrm{n}=$ 343) for comparison of the CFQ scores [13].

This study was performed in accordance with the Declaration of Helsinki and its amendments. The protocol was approved by the local medical ethics board of the Maastricht University Medical Center. Written informed consent for participation in this study was obtained from all subjects.

\section{Clinical Data}

Relevant clinical data including treatment, lung function measurements and chest radiographs were obtained. With respect to the use of medication during follow-up, patients with sarcoidosis were categorized into three treatment groups: (1) no immunomodulating drugs; (2) prednisone with or without methotrexate (MTX), and (3) anti-TNF- $\alpha$ drugs. Furthermore, it was recorded whether patients received medication at baseline. Forced expiratory volume in $1 \mathrm{~s}$ and forced vital capacity were measured with a pneumotachograph (Masterlab, Jaeger, Würzburg, Germany). Diffusing capacity for carbon monoxide was measured by the single-breath method on the Masterlab (Jaeger). Values are expressed as a percentage of predicted values.

Chest radiographs were graded according to the radiographic staging of DeRemee (0-III), adding stage IV: with signs of pulmonary fibrosis, loss of volume, hilar retraction and bullae. Two groups were distinguished: the first group included stages 0 and I, and the second stages II-IV.

\section{Questionnaires}

The sarcoidosis patients completed the CFQ developed by Broadbent et al. [11] and the Fatigue Assessment Scale (FAS) $[14,15]$ (http://www.ildcare.eu/index.php?id=100), the Center for Epidemiological Studies-Depression Scale (CES-D) [16], and the Small Fiber Neuropathy Screening List (SFNSL) [17] (http://www. ildcare.eu/index.php?id=100).

The CFQ is a self-report questionnaire consisting of 25 items assessing impairment in attention, perception, memory and motor functioning in everyday life [11]. In addition to these 25 items, all participants indicated on a 5 -point Likert scale if they had experienced an increase in cognitive failures in the past 5 years (termed 'increase', ranging from 'no increase' score 1, to 'very strong increase' score 5), and to what extent these cognitive impairments affected their daily life, ranging from 'no hindrance at all' (score 1) to 'very much hindrance' (score 5), how worried they were about these cognitive failures, ranging from 'not worried at all' (score 1) to 'very much worried' (score 5) and finally how annoying they found these cognitive failures, ranging from 'not annoying at all' (score 1) to 'very much annoying' (score 5). The total CFQ score was calculated by summing up all items, with the total 
score ranging from $0-100$. A high CFQ score was defined as a score $\geq 43$ (mean of the controls plus one standard deviation).

Statistical Analysis

Differences in mean scores between two groups were compared using Student's t test for independent samples and differences in mean scores between more than two groups were compared using analysis of variance. The proportions of patients with a high CFQ score within the sarcoidosis and control group were compared using the $\chi^{2}$ test.

Within the group of patients with sarcoidosis, the difference between the baseline score and the score at the end of the 6-month follow-up was calculated for both questionnaires (CFQ and FAS). The baseline score was subtracted from the follow-up score. Decreases in CFQ and FAS scores indicate decreased cognitive failure and fatigue and thus improvement. For the total group of patients with sarcoidosis, the one-sample $t$ test was used to test whether there was a significant change in scores during follow-up. In order to evaluate the effect of different types of medication on the CFQ score, mean changes between baseline and follow-up scores in the two treatment groups treated with immunomodulating drugs during the follow-up were compared with a reference group of patients without immunomodulating drug treatment using Student's t test for independent samples.

In order to evaluate whether treatment affected the change in the CFQ score that is independent of changes in self-reported fatigue, a multivariate linear regression model was used with change in CFQ score as dependent variable. In this model, treatment group was coded by two dummy variables, using the group who received no immunomodulating drugs as reference group. Change in FAS score was coded as a continuous variable. Statistical analyses were performed using SPSS (version 15.0) for Windows. A two-sided $\mathrm{p} \leq 0.05$ indicated statistical significance.

\section{Results}

The CFQ data of the sarcoidosis population and the matched control group are summarized in table 1 . The mean CFQ total score was significantly higher in sarcoidosis patients $(37.3 \pm 16.1)$ than in the controls (31.3 \pm 10.1; $\mathrm{p}<0.0001)$. More than one third of the patients had a high CFQ score (54.0 \pm 8.8$)$ versus $14.3 \%$ of the matched controls. Moreover, patients experienced significantly higher increases in cognitive failures in the past 5 years and were more hindered, annoyed and worried about these cognitive failures.

The demographic characteristics of the sarcoidosis population are summarized in table 2. Patients were not suffering from anemia. No relation was found with serum calcium levels, electrolytes or inflammatory parameters: C-reactive protein, angiotensin-converting enzyme or soluble interleukin 2 receptor. Moreover, lung function test results, arterial oxygen levels (at rest) and dyspnea did not correlate with the CFQ scores (data not shown) and
Table 1. Characteristics of sarcoidosis patients (cases, $n=343$ ) and matched controls $(n=343)$ at baseline

\begin{tabular}{lccc}
\hline & Cases & Controls [13] & p value \\
\hline Females, \% & 44.6 & 44.6 & NS \\
Age, years & $49.3 \pm 11.0$ & $45.5 \pm 11.0$ & NS \\
$\quad$ Range & $20-79$ & $24-79$ & \\
CFQ total score & $37.3 \pm 16.1$ & $31.3 \pm 10.1$ & $<0.0001$ \\
CFQ score $\geq 43, \mathrm{n}(\%)$ & $120(35.0)$ & $49(14.3)$ & $<0.0001$ \\
Additional questions: & & & \\
$\quad$ Increase & $2.7 \pm 1.1$ & $1.7 \pm 0.8$ & $<0.0001$ \\
$\quad$ Impairment & $2.8 \pm 1.1$ & $2.0 \pm 0.8$ & $<0.0001$ \\
$\quad$ Worry & $2.5 \pm 1.0$ & $1.8 \pm 0.9$ & $<0.0001$ \\
$\quad$ Annoyance & $2.6 \pm 1.2$ & $1.9 \pm 0.9$ & $<0.0001$ \\
\hline
\end{tabular}

Data are expressed as absolute numbers and means \pm SD (\%). NS $=$ Not significant

no differences were found in lung function test results between patients with a high CFQ compared to those with a normal CFQ score (table 2) at baseline. Of the studied patients, $70.6 \%$ had involvement of the lungs, $67.1 \%$ of lymph nodes; $34.5 \%$ of the eyes; $32.2 \%$ of the joints; $9.2 \%$ had cardiac involvement; $5.9 \%$ had signs of neurosarcoidosis and $11.4 \%$ had other manifestations of sarcoidosis; $16 \%$ of the patients had only extrathoracic signs of sarcoidosis. No differences regarding these mentioned clinical data were found between those patients with a high or normal CFQ score.

In the group with high CFQ scores, the number of women was significantly higher than in the group with normal CFQ scores, and this difference was also found in the healthy control group. No substantial differences were found in clinical data, including disease presentation, severity and duration, and lung function test results, between patients with a normal or a high CFQ score (table 2). At baseline, $42.3 \%(\mathrm{n}=145)$ of the patients with sarcoidosis used no immunomodulating drugs (no treatment), 37.3\% used prednisone (daily dose $9.1 \pm 7.5 \mathrm{mg}$; range: $1-60)$, and $20.4 \%$ used prednisone and MTX (10.4 $\pm 5.5 \mathrm{mg}$ once a week; range: $5-20)$. Thus, $57.7 \%(\mathrm{n}=198)$ of the patients were treated. Moreover, $35 \%$ of the cases received pain killers (non-steroidal anti-inflammatory drugs but none of the patients used opiates); $6.3 \%$ antidepressant drugs, and $8.2 \%$ sleep medication. None of the patients received treatment for cognitive problems. At baseline, the percentages of patients receiving treatment were similar for the group with high and normal CFQ scores (table 2). 
Table 2. Characteristics of the total sarcoidosis population subdivided by normal and high CFQ scores at baseline

\begin{tabular}{llccc}
\hline & $\begin{array}{l}\text { Total population } \\
(\mathrm{n}=343)\end{array}$ & $\begin{array}{l}\mathrm{CFQ}<43 \\
(\mathrm{n}=222)\end{array}$ & $\begin{array}{l}\text { CFQ } \geq 43 \\
(\mathrm{n}=121)\end{array}$ & p value \\
\hline Females, \% & 44.6 & 37.7 & 57.5 & $<0.0001$ \\
Age, years & $49.3 \pm 11.0$ & $50.3 \pm 11.3$ & $47.6 \pm 10.5$ & 0.50 \\
Time since diagnosis, years & $5.0 \pm 7.5(0-41)$ & $5.4 \pm 8.3$ & $4.3 \pm 5.5$ & 0.75 \\
DLCO, \% of predicted & $81.8 \pm 18.1$ & $82.4 \pm 18.8$ & $80.9 \pm 16.8$ & 0.50 \\
FVC, \% of predicted & $98.8 \pm 19.8$ & $98.0 \pm 20.7$ & $100.4 \pm 18.1$ & 0.30 \\
Chest X-ray stage: 0-I/II-IV, \% & $45.5 / 54.5$ & $43.0 / 57.0$ & $50.0 / 50.0$ & 0.22 \\
Treatment: no/yes, \% & $42.3 / 57.7$ & $40.8 / 59.2$ & $45.0 / 55.0$ & 0.55 \\
CFQ & $37.3 \pm 16.1$ & $27.3 \pm 10.0$ & $54.1 \pm 8.6$ & $<0.0001$ \\
FAS & $29.2 \pm 8.4$ & $26.6 \pm 7.8$ & $34.3 \pm 7.0$ & $<0.0001$ \\
CES-D & $13.9 \pm 9.0$ & $10.8 \pm 7.2$ & $19.9 \pm 9.2$ & $<0.0001$ \\
SFNSL & $42.9 \pm 7.3$ & $39.2 \pm 14.3$ & $49.7 \pm 20.3$ & $<0.0001$ \\
\hline
\end{tabular}

Data are expressed as means \pm SD or percentages (ranges). DLCO $=$ Diffusing capacity for carbon monoxide; FVC = forced vital capacity. Differences were evaluated between patients with CFQ $<43$ vs. $\geq 43$.

Table 3. Results (means \pm SD) with respect to CFQ and FAS scores according to treatment categories

\begin{tabular}{|c|c|c|c|c|c|}
\hline & $\begin{array}{l}\text { Group } 1 \\
(\mathrm{n}=189)\end{array}$ & $\begin{array}{l}\text { Group } 2 \\
(n=93)\end{array}$ & $\begin{array}{l}\text { Group } 3 \\
(\mathrm{n}=42)\end{array}$ & $\mathrm{p}$ value & $\begin{array}{l}\text { Total population } \\
(\mathrm{n}=324)\end{array}$ \\
\hline Females, $\%$ & 49.2 & 37.6 & 38.1 & NS & 44.6 \\
\hline \multicolumn{6}{|l|}{ CFQ total score } \\
\hline At baseline & $37.7 \pm 16.9$ & $34.7 \pm 13.7$ & $44.3 \pm 15.3$ & 0.004 & $37.7 \pm 15.7$ \\
\hline At 6 months & $37.6 \pm 17.2$ & $36.3 \pm 14.4$ & $37.2 \pm 14.7$ & NS & $37.2 \pm 16.1$ \\
\hline \multicolumn{6}{|l|}{ CFQ score $\geq 43$} \\
\hline At baseline & 36.0 & 31.2 & 59.5 & 0.005 & 37.6 \\
\hline At 6 months & 34.9 & 35.5 & 33.3 & NS & 34.5 \\
\hline$\Delta \mathrm{CFQ}$ & $-0.08 \pm 9.35$ & $1.67 \pm 9.22$ & $-7.07 \pm 7.23$ & $<0.0001$ & $-0.49 \pm 9.42$ \\
\hline \multicolumn{6}{|l|}{ FAS score } \\
\hline At baseline & $28.6 \pm 7.94$ & $28.2 \pm 7.81$ & $32.8 \pm 7.31$ & 0.004 & $29.0 \pm 7.93$ \\
\hline At 6 months & $29.0 \pm 7.59$ & $29.4 \pm 7.35$ & $27.9 \pm 6.56$ & NS & $29.0 \pm 7.39$ \\
\hline$\Delta$ FAS & $0.44 \pm 5.13$ & $1.19 \pm 4.87$ & $-4.90 \pm 5.57$ & $<0.0001$ & $-0.04 \pm 5.45$ \\
\hline
\end{tabular}

Group 1 = Untreated patients (no prednisone and/or MTX); group 2 = patients treated with prednisone with or without MTX; group 3 = patients treated with anti-TNF- $\alpha$ drugs; NS = not significant. At 6 months, $94.5 \%$ of 343 cases $(n=324)$ returned the questionnaire.

After 6 months of follow-up, the effect of treatment was evaluated. The reason for initiating treatment hereafter was never cognitive failure. Only the effect of the various treatments on cognitive functioning was evaluated. At baseline and after 6 months, 324 cases completed the questionnaires: 189 of them were not treated with either prednisone and/or MTX during this 6-month fol- low-up period (group 1). Group 2 consisted of 93 patients, of which 48 used corticosteroids only and 45 used low-dose corticosteroids together with MTX and folic acid. Group 3 comprised 42 of the 324 patients who were treated with anti-TNF- $\alpha$ drugs during these 6 months [infliximab $5 \mathrm{mg} / \mathrm{kg}$ every 4 weeks $(\mathrm{n}=31)$ or adalimumab $40 \mathrm{mg}$ s.c. once a week $(\mathrm{n}=11)]$. At baseline, this 
latter group, suffering from refractory sarcoidosis, used prednisone with MTX but did not demonstrate clinical improvement after the initially started therapy. After starting anti-TNF- $\alpha$ treatment this co-medication was reduced to, either to $7.5 \mathrm{mg}$ MTX once a week or to $5 \mathrm{mg}$ prednisone once a day (in case they developed side effects during MTX treatment), to avoid antibody formation.

Table 3 shows that for the total group of patients with sarcoidosis, the mean change in CFQ and FAS scores during follow-up was very small and not significant. However, when patients were categorized according to treatment (groups 1-3), the group of patients who received anti-TNF- $\alpha$ therapy during the follow-up period (group 3), showed substantial improvement in both CFQ and FAS scores. This change in scores is significantly higher than in the reference group of untreated patients (group 1) and the patients who were treated with prednisone with or without MTX (group 2) during the 6-month follow-up ( $\mathrm{p}<0.0001$; table 3$)$. After restriction to only those patients with a high CFQ score at baseline $(\mathrm{n}=121)$, the results remained similar and significant $(\mathrm{p}<0.0001)$. Improvement was still much higher in the group treated with anti-TNF- $\alpha$ drugs [group $1(\mathrm{n}=67$; $\Delta \mathrm{CFQ}$ : -1.75 ; $\Delta$ FAS: 0.59); group $2(\mathrm{n}=29 ; \Delta \mathrm{CFQ}:-0.86 ; \Delta \mathrm{FAS}: 0.86)$, and group 3 ( $\mathrm{n}=25$; $\triangle \mathrm{CFQ}$ : $8.48 ; \Delta \mathrm{FAS}:-5.36)$ ]. After adjustment for change in FAS score using a multivariate linear regression model, the change in the CFQ score in the group of patients treated with anti-TNF- $\alpha$ drugs remained significant and higher than in the other groups. The change from baseline to 6 months was -4.94 (95\% confidence interval: -8.10 to -1.79$)$ compared with nonsignificant changes of -0.25 (95\% confidence interval: -1.53 to 1.02$)$ and 1.46 (95\% confidence interval: -0.76 to 3.69) in the reference group without any treatment and the group treated with prednisone with or without MTX, respectively.

\section{Discussion}

This study is the first to demonstrate that everyday cognitive failure is a substantial problem in sarcoidosis patients. This is remarkable, because sarcoidosis predominantly affects young adults. A high CFQ sore $(\geq 43)$ occurred in more than one third of the sarcoidosis patients compared with $14.3 \%$ in matched healthy controls. Patients experienced more failures in the past 5 years compared to controls. Moreover, these failures had more (emotional) effects on the patients; patients were signifi- cantly more hindered, annoyed and worried about their cognitive failures. No substantial relationship was found with disease presentation, severity or duration. Although the primary reason for initiating treatment was not cognitive failure but other sarcoidosis-related symptoms, only treatment with anti-TNF- $\alpha$ drugs resulted in an improvement in cognitive functioning, whereas untreated patients and patients treated with corticosteroids with or without MTX did not show progress in cognitive functioning. Moreover, the results indicate that a low cognitive performance (high CFQ score) is associated with more fatigue, depressive symptoms and symptoms related to small fiber neuropathy.

The findings in the present study raise the question of whether the higher frequency of cognitive impairment in patients with sarcoidosis can be explained by the higher frequency of fatigue, depression and symptoms related to small fiber neuropathy or whether the strong concordance between all these symptoms and cognitive impairment can be explained by a common underlying mechanism. Strong support for the latter explanation is given by the finding that treatment with anti-TNF- $\alpha$ drugs resulted in an improvement in the CFQ and FAS scores and that this treatment effect on the CFQ score was independent of the improvement in the FAS score. After adjustment for change in FAS score using a multivariate linear regression model, the change in the CFQ score in the group of patients treated with anti-TNF- $\alpha$ drugs remained high and significant. Therefore, it is hypothesized that the common underlying mechanism is associated with an increase in TNF- $\alpha$ levels. The most widely held view is that chronic disorders including sarcoidosis cause changes in the central nervous system (CNS) through possible processes such as vascular disease due to inflammation, including an increase in TNF- $\alpha$, oxidative stress and inflammation-induced changes in neurotransmitter metabolism $[18,19]$. Such CNS changes then lead to a lower cognitive function. Autonomic dysfunction and fatigue are both a dominant symptom of and risk factor for depression, and the conditions may share several neurobiological abnormalities, for example an increase in TNF- $\alpha$.

There is evidence that TNF- $\alpha$ regulates synaptic transmission in the brain and that this cytokine is involved in the spatial memory impairment in mice [20]. It is also assumed that neuroinflammation with overexpression of cytokines is a standard characteristic of the brain pathology present in Alzheimer's disease. Tobinick and Gross [21] reported rapid cognitive improvement in Alzheimer's disease following treatment with etanercept, which 
binds TNF- $\alpha$ and blocks its interaction with cell surface TNF- $\alpha$ receptors.

Recent evidence also suggests that TNF- $\alpha$ may induce the expression of inducible nitric oxide synthase (iNOS). Overexpression of iNOS was found in the brains of Alzheimer's disease patients. As a strategy to prevent the cognitive decline in Alzheimer's disease, anti-oxidants are well recommended based on the association of oxidative damage with the progress of the disease [18,22]. More recently, the TNF- $\alpha$ inhibitor thalidomide was found to be effective in reducing the iNOS/peroxynitrite-related pathology via restraining the increase in TNF- $\alpha$ without eventually harming the physiological function of iNOS [22]. Involvement of excess TNF- $\alpha$ in the pathogenesis of cognitive impairment and fatigue in patients with sarcoidosis would explain the favorable effect of anti-TNF- $\alpha$ drugs in this study.

The present study also draws attention to the high prevalence of everyday cognitive failure in patients with sarcoidosis. The results stress that sarcoidosis patients are at a higher risk for developing cognitive problems compared to healthy controls. In many chronic disorders it is well known that the decline in cognitive performance is likely to have important clinical consequences [1]. In sarcoidosis patients, this is even more important as this disorder affects mainly young adults. In this part of their lives, cognitive abilities are necessary for personal health, their social lives and maintenance of their abilities to function appropriately at work and to finish their studies successfully, for example. Providing effective medication for the treatment of cognitive deficits in general can have a dramatic effect on the quality of life of a patient dealing with this problem.

In sarcoidosis, inflammation results in an increase in TNF- $\alpha$ [23]. In the present study, anti-TNF- $\alpha$ treatment appeared beneficial in reducing cognitive failure, as well as disease activity and fatigue. Two randomized, doubleblind, placebo-controlled trials have been performed in pulmonary sarcoidosis. Baughman et al. [24] found a significant improvement in the forced vital capacity of patients receiving infliximab compared to placebo, in agreement with Rossman et al. [25]. More recently, Moravan and Segal [26] demonstrated that combination treatment with mycophenolate mofetil and infliximab is a promising therapeutic approach for refractory neurosarcoidosis. Case reports suggest that this agent can be beneficial in patients with neurosarcoidosis and small-fiber neuropathy [27]. Also, thalidomide treatment was effective in sarcoidosis [28]. However, cognition was beyond the scope of these studies and not evaluated.
One of the limitations of this observational study was that the neuropsychological assessment for cognitive dysfunctions was restricted to a rather global and subjective screening instrument. Labeling oneself as absent-minded or forgetful depends upon the perceived discrepancy between the subject's everyday memory functioning and her/his everyday memory demands. Selfreported cognitive changes or decline do not necessarily reflect actual cognitive decline. Beliefs about cognitive changes are strongly influenced by self-efficacy beliefs, personality, vitality and coping styles [29]. This influence might explain the higher proportion of women with high CFQ scores in both the sarcoidosis group and the matched control group. Future studies using objective measures of cognitive performance to estimate the prevalence of everyday cognitive impairment in patients with sarcoidosis are recommended. Nevertheless, it seems unlikely that the favorable effect on cognitive function reported by patients who were treated with anti-TNF- $\alpha$ drugs is attributable to the use of a subjective screening instrument.

Another limitation of the study was that it was not feasible to evaluate whether or not the improvement in CFQ after anti-TNF- $\alpha$ treatment was direct or mediated through decreases in depressive symptoms. Patients completed the CES-D questionnaire at baseline, but results on CES-D scores at 6 months were not available. Both depression and fatigue are very important issues regarding cognition. Moreover, we found that autonomic dysfunction is also involved. However, in our population fatigue appeared the most prominent of these three factors. Therefore, we decided to evaluate fatigue, next to cognition, after the 6-month follow-up (table 3). One of the main reasons for this was to prevent patients refusing to participate in the second round because they got the impression that they needed to complete too many questionnaires. Our experience is that patients are willing to participate, if this is not too time consuming. The relationship between depression and cognition should be addressed in further studies. Furthermore, there might be a concern that improvement in cognition could be associated with improvement in lung function test results. However, at baseline, no association was found between lung function and cognitive failure. Patients with cognitive failure had either normal lung function or moderately decreased values. These lung function test results were comparable with those of patients without signs of cognitive failure. 


\section{Conclusions}

In conclusion, everyday cognitive failure is a substantial problem in patients with sarcoidosis. More than one third of the sarcoidosis patients exhibited cognitive deficits compared to $14.3 \%$ of healthy volunteers. Our study should raise awareness that sarcoidosis patients, similar to patients with other chronic disorders, are at higher risk for developing cognitive impairment. This will likely further worsen their general health. Anti-TNF- $\alpha$ treatment was found to be successful in the treatment of refractory sarcoidosis. It not only reduced fatigue but, interestingly, also had a positive effect on the cognitive functioning of sarcoidosis patients. These results emphasize the need for further research to integrate knowledge about neuropsychological deficits, coping, cognitive failure and cognitive performance, fatigue, depressive symptoms and treatment strategies in sarcoidosis into clinical management. These findings render managing sarcoidosis patients even more challenging.

\section{Appendix}

Items of the CFQ [11]

(1) Do you read something and find you haven't been thinking about it and must read it again?

(2) Do you find you forget why you went from one part of the house to the other?

(3) Do you fail to notice signposts on the road?

(4) Do you find you confuse right and left when giving directions?

(5) Do you bump into people?

(6) Do you find you forget whether you've turned off a light or a fire or locked the door?

(7) Do you fail to listen to people's names when you are meeting them?

(8) Do you say something and realize afterwards that it might be taken as insulting?

(9) Do you fail to hear people speaking to you when you are doing something else?

(10) Do you lose your temper and regret it?

(11) Do you leave important letters unanswered for days?

(12) Do you find you forget which way to turn on a road you know well but rarely use?

(13) Do you fail to see what you want in a supermarket (although it's there)?
(14) Do you find yourself suddenly wondering whether you've used a word correctly?

(15) Do you have trouble making up your mind?

(16) Do you find you forget appointments?

(17) Do you forget where you put something like a newspaper or a book?

(18) Do you find you accidentally throw away the thing you want and keep what you meant to throw away - as in the example of throwing away the matchbox and putting the used match in your pocket?

(19) Do you daydream when you ought to be listening to something?

(20) Do you find you forget people's names?

(21) Do you start doing one thing at home and get distracted into doing something else (unintentionally)?

(22) Do you find you can't quite remember something although it's 'on the tip of your tongue'?

(23) Do you find you forget what you came to the shops to buy?

(24) Do you drop things?

(25) Do you find you can't think of anything to say?

Online form: http://www.ildcare.eu/index.php?id=100.

\section{References}

1 Hung WW, Wisnivesky JP, Siu AL, Ross JS: Cognitive decline among patients with chronic obstructive pulmonary disease. Am J Respir Crit Care Med 2009;180:134-137.

2 Bargagli E, Maggiorelli C, Rottoli P: Human chitotriosidase: a potential new marker of sarcoidosis severity. Respiration 2008;76: $234-238$

3 Handa T, Nagai S, Hirai T, Chin K, Kubo T, Oga T, Niimi A, Matsumoto H, Ito Y, Takahashi K, Watanabe K, Izumi T, Mishima M: Computed tomography analysis of airway dimensions and lung density in patients with sarcoidosis. Respiration 2009;77:273-281.
4 Rothkrantz-Kos S, van Dieijen-Visser MP, Mulder PG, Drent M: Potential usefulness of inflammatory markers to monitor respiratory functional impairment in sarcoidosis. Clin Chem 2003;49:1510-1517.

5 Wijnen PA, Nelemans PJ, Verschakelen JA, Bekers O, Voorter CE, Drent M: The role of tumor necrosis factor alpha G-308A polymorphisms in the course of pulmonary sarcoidosis. Tissue Antigens 2010;75:262-268.

6 Iannuzzi MC, Rybicki BA, Teirstein AS: Sarcoidosis. N Engl J Med 2007;357:2153-2165.
7 Drent M, Wirnsberger RM, Breteler $\mathrm{MH}$, Kock LM, de Vries J, Wouters EF: Quality of life and depressive symptoms in patients suffering from sarcoidosis. Sarcoidosis Vasc Diffuse Lung Dis 1998;15:59-66.

8 Wirnsberger RM, de Vries J, Breteler MH, van Heck GL, Wouters EF, Drent M: Evaluation of quality of life in sarcoidosis patients. Respir Med 1998;92:750-756.

$\checkmark 9$ Cox CE, Donohue JF, Brown CD, Kataria YP, Judson MA: Health-related quality of life of persons with sarcoidosis. Chest 2004;125: 997-1004. 
10 Chang B, Steimel J, Moller DR, Baughman RP, Judson MA, Yeager H Jr, Teirstein AS, Rossman MD, Rand CS: Depression in sarcoidosis. Am J Respir Crit Care Med 2001; 163:329-334.

-11 Broadbent DE, Cooper PF, FitzGerald P, Parkes KR: The Cognitive Failures Questionnaire (CFQ) and its correlates. Br J Clin Psychol 1982;21:1-16.

-12 Statement on sarcoidosis. Joint Statement of the American Thoracic Society (ATS), the European Respiratory Society (ERS) and the World Association of Sarcoidosis and Other Granulomatous Disorders (WASOG) adopted by the ATS Board of Directors and by the ERS Executive Committee, February 1999. Am J Respir Crit Care Med 1999;160:736755.

13 van Boxtel MP, Buntinx F, Houx PJ, Metsemakers JF, Knottnerus A, Jolles J: The relation between morbidity and cognitive performance in a normal aging population. J Gerontol A Biol Sci Med Sci 1998;53:M147M154.

14 Michielsen HJ, De Vries J, Drent M, PerosGolubicic T: Psychometric qualities of the Fatigue Assessment Scale in Croatian sarcoidosis patients. Sarcoidosis Vasc Diffuse Lung Dis 2005;22:133-138.

- 15 De Vries J, Michielsen H, Van Heck GL, Drent M: Measuring fatigue in sarcoidosis: the Fatigue Assessment Scale (FAS). Br J Health Psychol 2004;9:279-291.
16 Radloff L: The CES-D scale: A self-report depression scale for research in the general population. Appl Psychol Meas 1977;1:385401.

17 Hoitsma E: Small Fiber Neuropathy. A Novel Finding in Sarcoidosis; thesis. Maastricht: University of Maastricht, 2005.

18 McAfoose J, Baune BT: Evidence for a cytokine model of cognitive function. Neurosci Biobehav Rev 2009;33:355-366.

19 Hoitsma E, Faber CG, Drent M, Sharma OP: Neurosarcoidosis: a clinical dilemma. Lancet Neurol 2004;3:397-407.

20 Baune BT, Wiede F, Braun A, Golledge J, Arolt V, Koerner H: Cognitive dysfunction in mice deficient for TNF- and its receptors. Am J Med Genet B Neuropsychiatr Genet 2008;147B:1056-1064.

21 Tobinick EL, Gross H: Rapid cognitive improvement in Alzheimer's disease following perispinal etanercept administration. J Neuroinflammation 2008;5:2.

22 Alkam T, Nitta A, Mizoguchi H, Saito K, Seshima M, Itoh A, Yamada K, Nabeshima T: Restraining tumor necrosis factor-alpha by thalidomide prevents the amyloid beta-induced impairment of recognition memory in mice. Behav Brain Res 2008;189:100-106.

23 Baughman RP, Lower EE, Drent M: Inhibitors of tumor necrosis factor (TNF) in sarcoidosis: who, what, and how to use them. Sarcoidosis Vasc Diffuse Lung Dis 2008;25: $76-89$.
24 Baughman RP, Drent M, Kavuru M, Judson MA, Costabel U, du Bois R, Albera C, Brutsche M, Davis G, Donohue JF, MullerQuernheim J, Schlenker-Herceg R, Flavin S, Lo KH, Oemar B, Barnathan ES: Infliximab therapy in patients with chronic sarcoidosis and pulmonary involvement. Am J Respir Crit Care Med 2006;174:795-802.

$\checkmark 25$ Rossman MD, Newman LS, Baughman RP, Teirstein A, Weinberger SE, Miller W Jr, Sands BE: A double-blinded, randomized, placebo-controlled trial of infliximab in subjects with active pulmonary sarcoidosis. Sarcoidosis Vasc Diffuse Lung Dis 2006;23: 201-208.

26 Moravan M, Segal BM: Treatment of CNS sarcoidosis with infliximab and mycophenolate mofetil. Neurology 2009;72:337-340.

27 Hoitsma E, Faber CG, van Santen-Hoeufft M, De Vries J, Reulen JP, Drent M: Improvement of small fiber neuropathy in a sarcoidosis patient after treatment with infliximab. Sarcoidosis Vasc Diffuse Lung Dis 2006;23: 73-77.

28 Baughman RP, Judson MA, Teirstein AS, Moller DR, Lower EE: Thalidomide for chronic sarcoidosis. Chest 2002;122:227232.

29 Ponds RW, Commissaris KJ, Jolles J: Prevalence and covariates of subjective forgetfulness in a normal population in The Netherlands. Int J Aging Hum Dev 1997;45:207-221. 